\title{
Lentiviral gene transfer to reduce atherosclerosis progression by long-term CC-chemokine inhibition
}

\author{
CA Bursill ${ }^{1,4}$, E McNeill ${ }^{1,4}$, L Wang ${ }^{2}$, OC Hibbitt ${ }^{2}$, R Wade-Martins², DJ Paterson ${ }^{2}$, DR Greaves ${ }^{3}$ \\ and KM Channon ${ }^{1}$ \\ ${ }^{1}$ Department of Cardiovascular Medicine, John Radcliffe Hospital, Oxford, UK; ${ }^{2}$ Department of Anatomy, Physiology and Genetics, \\ University of Oxford, Oxford, UK and ${ }^{3}$ Sir William Dunn School of Pathology, Oxford, UK
}

\begin{abstract}
CC-chemokines are important mediators in the pathogenesis of atherosclerosis. Atherosclerosis progression is reduced by high-level, short-term inhibition of CC-chemokine activity, for example by adenoviral gene transfer. However, atherosclerosis is a chronic condition where short-term effects, while demonstrating proof-of-principle, are unlikely to provide maximum therapeutic benefit. Accordingly, we generated a recombinant lentivirus, lenti35K, encoding the broad-spectrum CC chemokine inhibitor, 35K, derived from the vaccinia virus. To investigate the effects of prolonged broad-spectrum chemokine inhibition on atherosclerosis, lenti35K, or lentiGFP or PBS were delivered to 6-week-old ApoE knockout (ApoE-KO) mice by hydrodynamic injection. Sustained lentiviral transduction and transgene expression were demonstrated by $35 \mathrm{~K}$ mRNA and viral DNA in liver tissue,
\end{abstract}

and recombinant $35 \mathrm{~K}$ protein circulating in the plasma, 3 months after gene transfer. Plasma from lenti35K animals had reduced chemokine activity compared with plasma from lentiGFP or PBS-treated animals. Histologic analysis of aortic sinus sections revealed that atherosclerotic plaque area in lenti35K mice was significantly reduced compared with both lentiGFP and PBS controls. Furthermore, plaque macrophage content was substantially reduced in lenti35K mice. Lentiviral $35 \mathrm{~K}$ gene transfer is a promising experimental strategy to reduce atherosclerosis progression, and demonstrates the potential of long-term CC-chemokine inhibition as a potential therapeutic target in atherosclerosis.

Gene Therapy (2009) 16, 93-102; doi:10.1038/gt.2008.141; published online 18 September 2008

Keywords: lentivirus; atherosclerosis; chemokine; hydrodynamic; ApoE

\section{Introduction}

Atherosclerosis, the underlying cause of heart attack and stroke, is a chronic inflammatory disease characterized by the accumulation of macrophages in atherosclerotic plaque. Atherosclerotic plaque progression leads to both chronic ischemia, related to plaque growth and to acute vascular events, related to plaque rupture and thrombosis. ${ }^{1}$ Chemokines (CKs; chemotactic cytokines), particularly the CC-CK class, have important functions in the pathogenesis of atherosclerosis, including regulation of monocyte recruitment and plaque growth, plaque composition and stability. ${ }^{2}$ CC-CKs including MCP-1, RANTES and MIP- $1 \alpha$ are present in human atherosclerotic lesions. ${ }^{3-6}$ In the atherosclerosis-prone apolipoprotein E knockout (ApoE-KO) mice, targeted deletion of single $\mathrm{CC}-\mathrm{CKs}$ or $\mathrm{CK}$ receptors significantly reduces atherosclerotic plaque progression ${ }^{7-9}$ demonstrating that a number of different CC-CK/CC-CK receptor interactions are important in atherosclerosis. Indeed, the marked functional redundancy in the $\mathrm{CK} / \mathrm{CK}$ receptor signalling system suggests that a more broad-spectrum approach to

Correspondence: Professor KM Channon, Department of Cardiovascular Medicine, John Radcliffe Hospital, Oxford OX3 9DU, UK.

E-mail: keith.channon@cardiov.ox.ac.uk

${ }^{4}$ These authors contributed equally to this work.

Received 14 January 2008; revised 28 April 2008; accepted 26 May 2008; published online 18 September 2008
CC-CK signaling may provide a more efficient strategy to reduce atherosclerosis.

The vaccinia virus (strain Lister) expresses a $35 \mathrm{KDa}$ protein ' $35 \mathrm{~K}$ ' that binds to and inactivates nearly all of the CC-CK class. ${ }^{10,11}$ Studies using an adenovirus vector engineered to express soluble $35 \mathrm{~K}$ (Ad35K) have demonstrated that short-term expression of $35 \mathrm{~K}$ in vivo significantly reduces atherosclerotic plaque in ApoE-KO mice. $^{12,13}$ These studies, using adenoviral gene transfer, provide important proof-of-principle that short-term, broad-spectrum CC-CK blockade can reduce atherosclerotic plaque in the early stages of atherosclerosis. However, modifying the long-term pathogenesis of atherosclerosis would likely require long-term CC-CK inhibition. Moreover, the effects of sustained broadspectrum CC-CK inhibition in atherosclerosis might be unpredictable. Specifically, the effects of CC-CK inhibition on signalling through MCP-1/CCR2 and RANTES/ CCR5 might be expected to inhibit plaque progression, whereas inhibition of CCL19/CCL21 might have the opposite effect, through blockade of macrophage emigration. ${ }^{14}$ Thus, the net effect of long-term, broadspectrum CC-CK blockade in atherosclerosis remains an important question to be addressed.

Modified lentivirus vectors can direct stable gene transfer by evasion of host immunosurveillance ${ }^{15-17}$ through incorporation into the host genome and absence of viral gene expression. ${ }^{18}$ However, efficient transduction rates in vivo are difficult to achieve, typically 
requiring delivery through the portal vein, ${ }^{19}$ injection into the facial vein of neonates ${ }^{16}$ or transplantation of transduced bone marrow. ${ }^{17}$ More efficient in vivo transduction by lentiviral vectors is required to provide prolonged gene transfer with biologically important effects. ${ }^{20}$

In this study, we use hydrodynamic intravenous delivery of lentivirus to achieve prolonged and efficient expression of the chemokine-binding protein $35 \mathrm{~K}$, to test the effects of broad-spectrum CC-CK inhibition on atherosclerosis in ApoE-KO mice.

\section{Results}

\section{Efficient and persistent transduction by lenti35K and lentiGFP in vitro}

We first tested the efficacy of transduction and transgene expression of lentiviral gene transfer in 293 cells in vitro. Cells transduced with lenti35K expressed soluble $35 \mathrm{~K}$ protein that was readily detectable in culture medium (Figure 1a), with expression persisting at both 4 and 8 weeks after transduction (Figure 1b). LentiGFP also transduced 293 cells efficiently in vitro. Green fluorescent cells were visualized following transduction by $1 \times 10^{3}-$ $1 \times 10^{1}$ TU of purified lentiGFP (Figure 1c). These cells remained green during continuous culture for 3 months.

\section{No toxicity of lentiviral gene transfer by hydrodynamic delivery}

To assess any potential toxicity on account of our novel strategy of administering lentiviral vectors by hydrodynamic delivery we assessed liver inflammation and serum liver transaminase levels. Mice received either phosphate-buffered saline (PBS) or lentiGFP by hydrodynamic delivery or adenoviral GFP (AdGFP) and were culled after 7 days along with untreated control animals.

We found no striking alterations in liver morphology or inflammatory infiltrate in H\&E stained sections from PBS- or lentiGFP-treated animals, compared with untreated controls (Figure 2a). However, in AdGFP-treated animals the livers showed a prominent inflammatory infiltrate and some necrosis. To look more closely for leukocyte influx related to either necrosis or viral immune response sections were stained with antiS100A9 to quantify neutrophil influx ${ }^{21}$ and anti-CD3 to assess T-cell influx (Figure 2a). No significant increase in liver neutrophil or T-cell content was seen following hydrodynamic PBS or lentiGFP treatment, compared with untreated control (Figure 2b). AdGFP caused a significant elevation of both cell types in the liver, indicating that this method of gene transfer of the same protein is more inflammatory than the lentiviral delivery.

To confirm a lack of liver toxicity following lentiGFP delivery serum liver transaminases, a cardinal sign of liver damage, were measured (Figure 2b). No significant elevation of either ALT or AST was found in the PBS or lentiGFP-treated animals, with AdGFP animals showing a significant elevation of both ALT and AST above levels in untreated control animals.

\section{Persistence of viral DNA and transgene expression after lentiviral gene transfer in vivo}

We next evaluated the efficacy and persistence of lentiviral gene transfer in vivo, using hydrodynamic delivery in mice. Using RT-PCR, 35K mRNA was detected 3 months following gene transfer in liver tissue samples from lenti35K-transduced animals (Figure 3a),
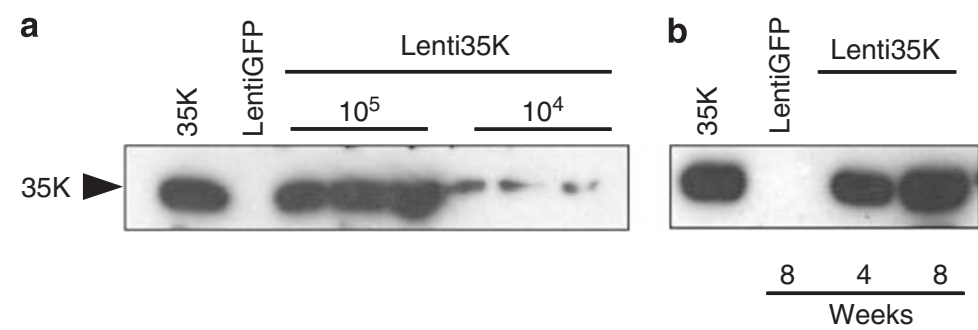

C $10^{3}$
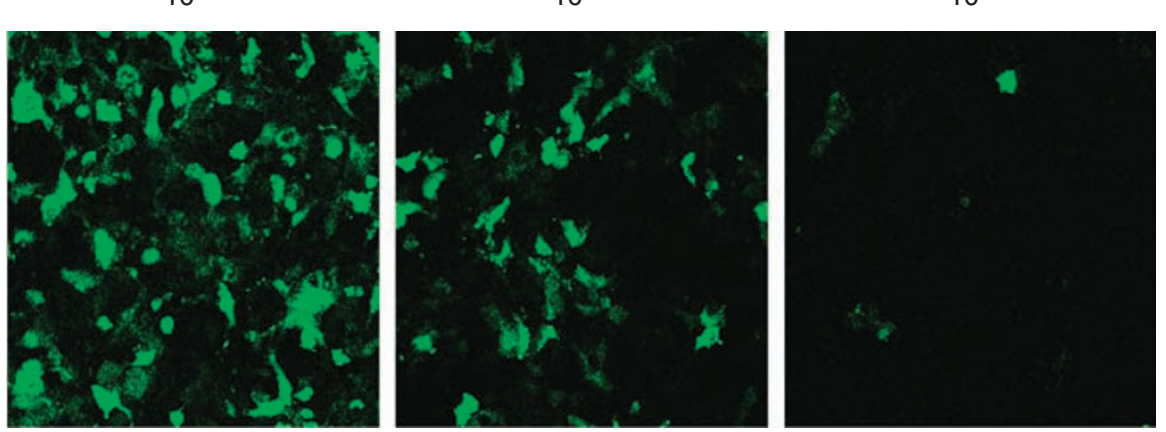

Figure 1 Cell transduction by lentivirus gene transfer in vitro. Purified lentiviruses were added to 293 cells at a range of titers $\left(1 \times 10^{5}-\right.$ $\left.1 \times 10^{1}\right)$ then incubated in the presence of polybrene $(8 \mu \mathrm{g} / \mathrm{ml})$ for $24 \mathrm{~h}$. Media $(10-20 \mu \mathrm{l})$ from lentiGFP and lenti35K-transduced cells were fractionated by SDS-PAGE, transferred to a membrane and assessed by western immunoblotting with rabbit polyclonal 35K antibody (1:2000 dilution). (a) Detection of 35K protein in the media of transduced cells after 1 week and (b) 4-8 weeks after transduction with lenti35K, using western blotting. (c) Green fluorescent cells from LentiGFP transductions were visualized by microscopy. These cells remained green for at least 3 months. Media from $35 \mathrm{~K}$ transfected cells is included in (a and $\mathbf{b}$ ) as a positive control for the western blot. 
a
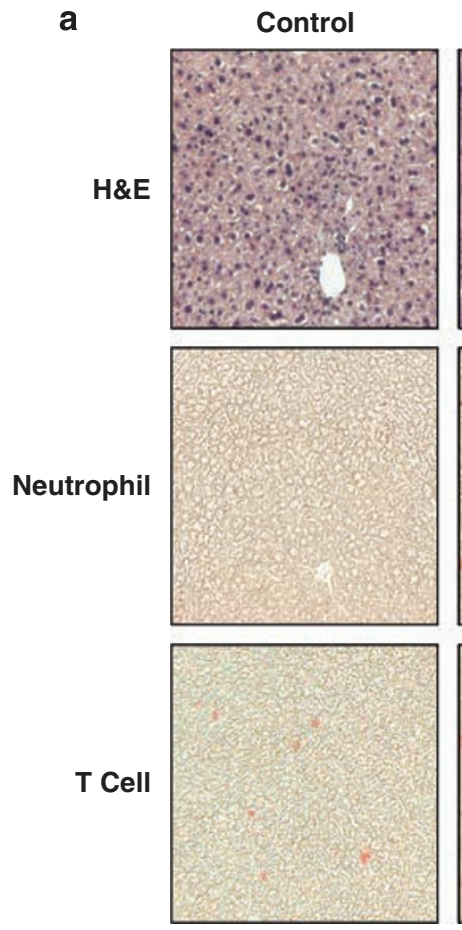

b

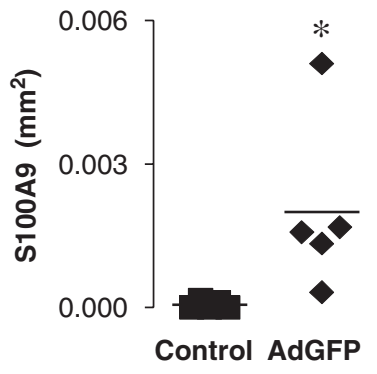

C

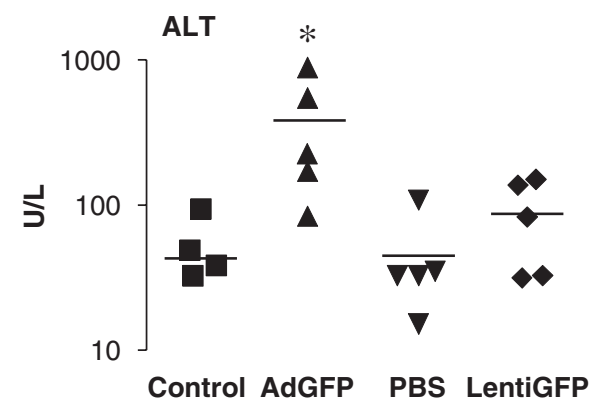

AdGFP
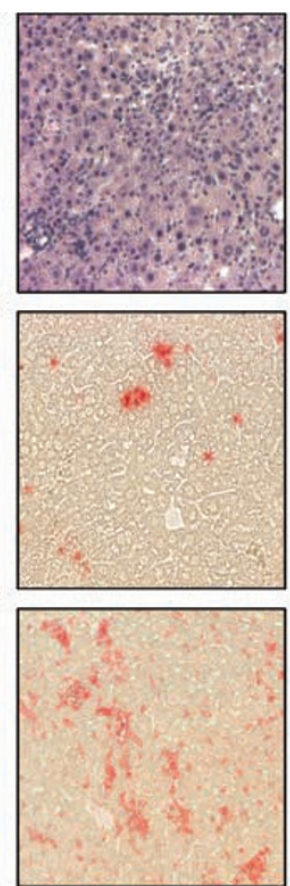

PBS
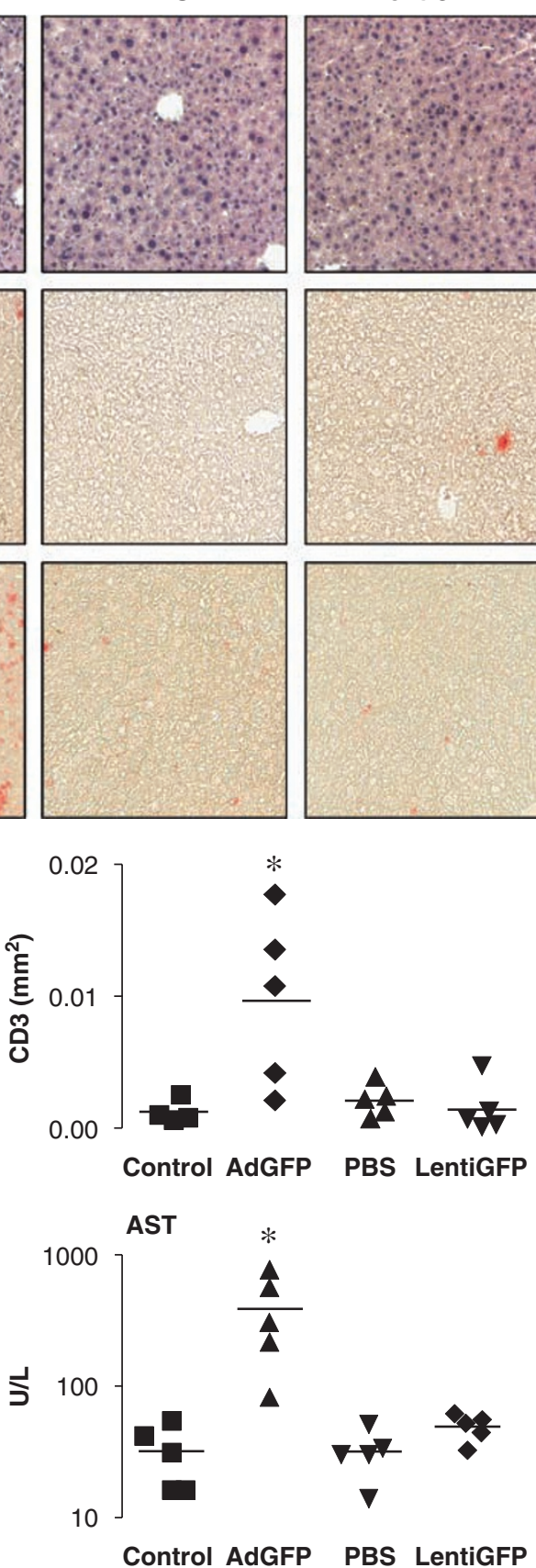

Figure 2 Hydrodynamic delivery of lentiviral vectors causes no significant liver toxicity or inflammation. ApoE-KO mice received PBS or lentiGFP through hydrodynamic injection, AdGFP by i.v. injection or no treatment at 10-12 weeks of age and were killed 7 days later. Paraffin-embedded liver sections were stained with hematoxylin and eosin, anti-S100A9 (neutrophils) or anti-CD3 (T cells) antibody. Representative sections are shown for each treatment group. (a) Quantification of the area of S100A9 or CD3 staining (per $10 \times$ field of view) shows no significant elevation of either cell type in lentiGFP or PBS-treated animals compared with untreated control. (b) Serum transaminase measurement shows no elevation of either ALT or AST in PBS or lentiGFP-treated animals. (c) As a control AdGFP caused both increased leukocyte infiltrate and elevated serum transaminases. *Denotes a significant difference between AdGFP and untreated control group, $P<0.05, n=5$ mice/treatment group.

but not from control animals, nor in samples without RT. We used quantitative Taq-Man RT-PCR to quantify the expression levels of $35 \mathrm{~K}$ and GFP in all animals 3 months after gene transfer. Transgene mRNA was detected at levels above the assay background (water control) exclusively in lenti35K or lentiGFP-transduced animals in the respective RT-PCR assay (Figure $3 \mathrm{~b}$ ). However, the relative level of the respective transgene expression was similar in both the lenti35K and lentiGFP groups.

To determine lentiviral vector persistence, we quantified viral DNA by PCR in mouse livers. Lenti35K viral DNA was detected in lenti35K-transduced mice 3 months after gene transfer (Figure 3c) but was not detected in either PBS or lentiGFP mouse liver DNA or 

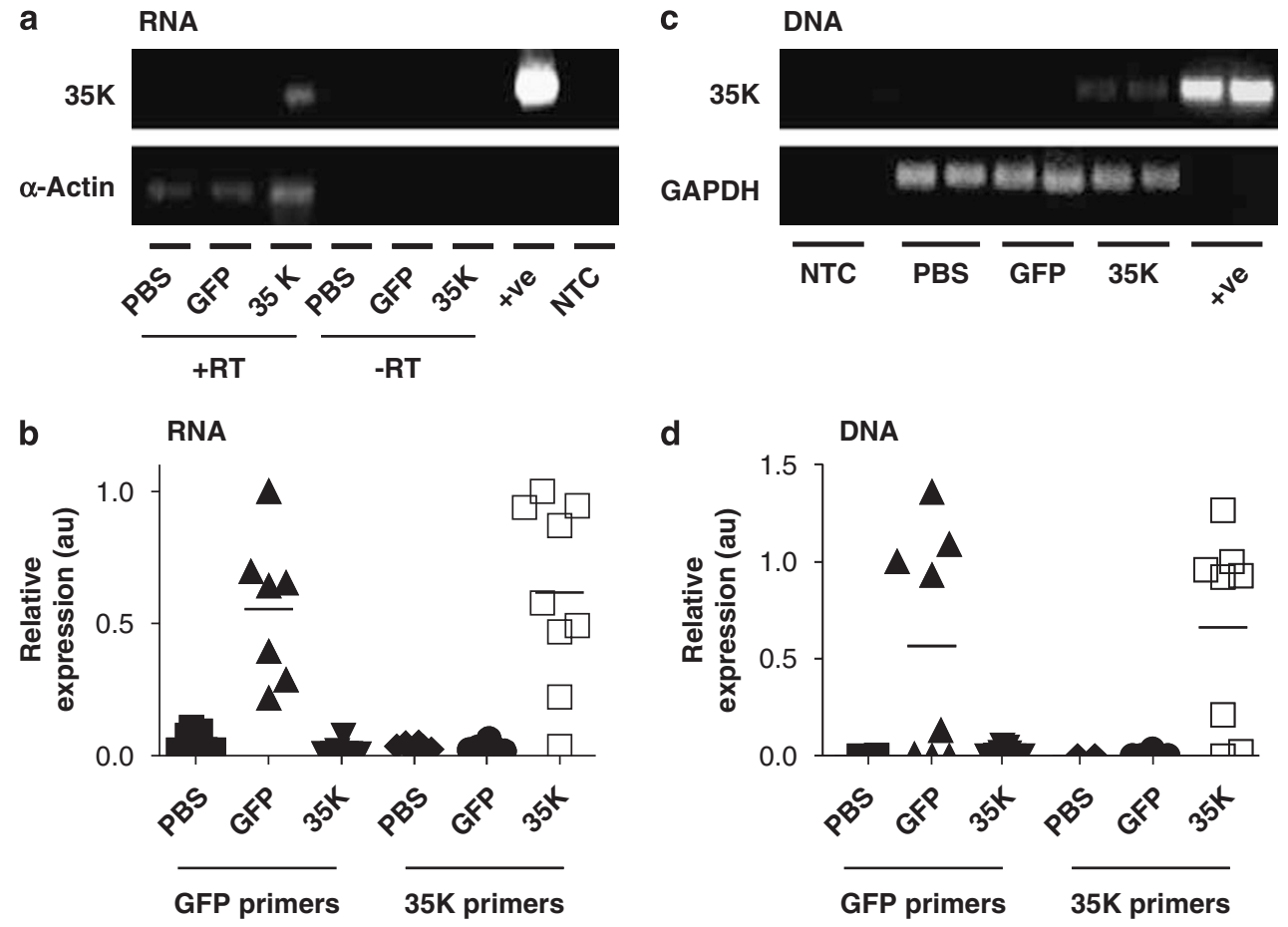

Figure 3 Detection of 35K and GFP mRNA and viral DNA in the livers of mice 3 months after gene transfer. ApoE-KO mice received PBS, lentiGFP or lenti35K at 6 weeks of age through hydrodynamic injection and were killed 3 months later. RNA and DNA were isolated from livers then $35 \mathrm{~K}$ and GFP expression or viral DNA was quantified using real-time PCR. (a) 35K mRNA was detected in RT-PCRs from lenti35K mice. (b) $35 \mathrm{~K}$ mRNA was detected 3 months after gene transfer exclusively in the lenti35K mouse livers and GFP mRNA was detected exclusively in lentiGFP livers. (c) 35K viral DNA was detected in PCRs from lenti35K mice. (d) $35 \mathrm{~K}$ viral DNA was detected 3 months after lentivirus gene transfer exclusively in the livers of mice injected with lenti35K and GFP viral DNA was detected exclusively in the livers of lentiGFP mice. '+ve' denotes the 35K plasmid control and 'NTC' denotes no template control in the RT-PCR experiment.

water control. Correspondingly, lentiGFP viral DNA was detected 3 months after gene transfer, exclusively in lentiGFP-transduced animals (Figure 3d).

\section{Protein expression and reduction of plasma CC-chemokine activity by lentiviral $35 \mathrm{~K}$ gene transfer in ApoE-KO mice}

We next evaluated the biological effects of in vivo hydrodynamic delivery of lenti35K on CC-CK activity in ApoE-KO mice. Twelve weeks after lenti35K delivery through hydrodynamic injection, $35 \mathrm{~K}$ protein was detected in plasma by western blotting (Figure 4a), although at levels approximately 10-fold lower than the very high levels observed in animals that had received adenoviral gene transfer of $35 \mathrm{~K}, 2$ weeks previously. No $35 \mathrm{~K}$ protein was detected in the plasma of control mice injected with either PBS or lentiGFP. Conversely, in lentiGFP-injected animals, microscopy of bone marrow cells 3 months after hydrodynamic delivery revealed green fluorescent cells that were not present in mice injected with either PBS or lenti35K (Figure $4 b$ ).

To evaluate the effect of $35 \mathrm{~K}$ expression on CCchemokine activity, we quantified the CCR5-directed cell migration evoked by plasma from mice that had received PBS, lenti35K or lentiGFP (Figure 4c). CCR5-directed cell migration was reduced by $\sim 60 \%$ in mice injected with lenti35K compared with the plasma of control mice injected with either PBS or lentiGFP. In contrast to the significant reduction in plasma CC-CK activity observed in lenti35K animals, no differences were detected in plasma concentrations of MCP-1 or RANTES between PBS, lentiGFP or lenti35K treatment groups (Table 1).

\section{Long-term CC-chemokine inhibition with $35 \mathrm{~K}$ reduces atherosclerosis in ApoE-KO mice}

To determine the effects of long-term CC-chemokine inhibition on atherosclerotic plaque progression, 6-weekold ApoE-KO mice received either PBS, lentiGFP or lenti35K by hydrodynamic delivery. There were no differences in plasma cholesterol concentrations between the PBS, lentiGFP or lenti35K groups (Table 1). After 3 months, atherosclerotic plaque area was quantified in paraffin-embedded aortic sinus sections (Figure 5). Longterm expression of $35 \mathrm{~K}$, significantly reduced atherosclerotic lesion size by approximately $60 \%$ compared with either PBS and lentiGFP control mice $(P<0.05)$. Furthermore, immunostaining with macrophage-specific antibodies mac3 and anti-Galectin- $3^{22}$ revealed that macrophage content in aortic sinus atherosclerotic plaques was strikingly reduced, by approximately $80 \%$, after lenti35K gene transfer (Figure 6), compared with both PBS and lentiGFP controls.

\section{Discussion}

In this study, we have demonstrated prolonged expression of a soluble, broad-spectrum recombinant CC-CK inhibitor protein, $35 \mathrm{~K}$, delivered by a single intravenous hydrodynamic injection of recombinant lentivirus. To investigate the effects of long-term CC-CK inhibition on 
a

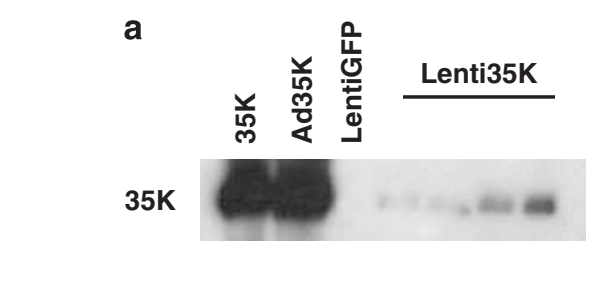

C

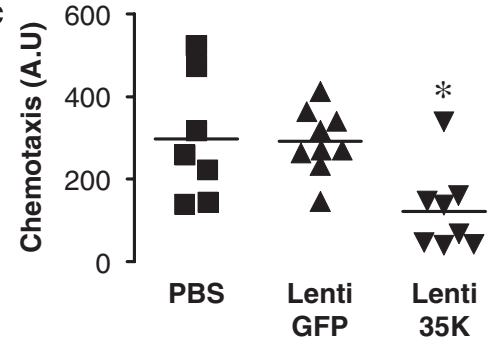

b

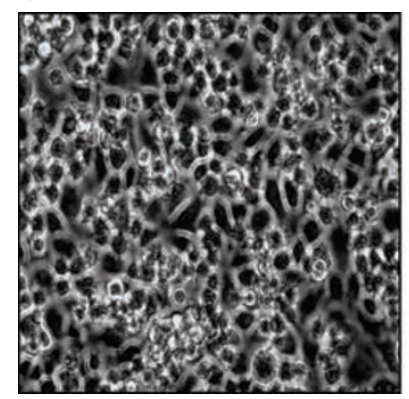

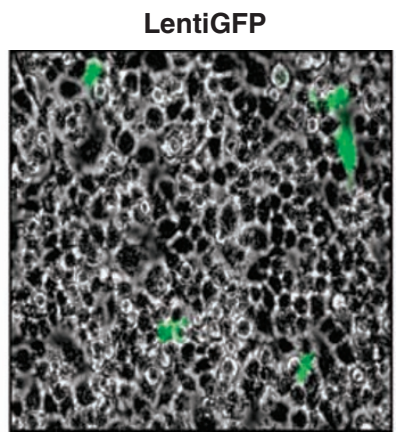

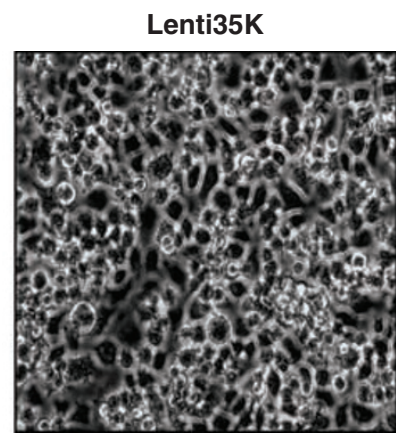

Figure 4 Detection of $35 \mathrm{~K}$ and GFP protein and quantification of chemokine activity in mice 3 months after gene transfer. ApoE-KO mice received PBS, lentiGFP or lenti35K at 6 weeks of age through hydrodynamic injection and were killed 3 months later. (a) Control and mouse plasma samples $(200 \mu \mathrm{l})$ were incubated with anti-HA agarose-conjugated beads and then fractionated by SDS-PAGE. 35K protein was detected in the plasma of mice 3 months after lenti35K gene transfer (lanes 4-7). A much stronger band was present from the plasma of a mouse injected with an adenovirus-expressing 35K (Ad35K, lane 2). No 35K protein was detected in lentiGFP mouse plasma (lane 3). (b) Detection of green fluorescent cells in the bone marrow of lentiGFP mice that are not present in PBS or lenti35K bone marrow. (c) In a Boyden chamber assay, plasma $(10 \mu \mathrm{l})$ from lenti35K mice induced significantly less CCR5-directed cell migration than plasma from PBS and lentiGFP mice. *Denotes a significant difference from PBS and lentiGFP control groups, $P<0.05, n=7-9$ mice/treatment group.

Table 1 Plasma chemokine and cholesterol concentrations

\begin{tabular}{lccc}
\hline & PBS & LentiGFP & Lenti35K \\
\hline $\begin{array}{l}\text { Chemokines: }\left(p g \mathrm{ml}^{-1}\right) \\
\quad \text { RANTES }\end{array}$ & $23.5 \pm 2.9$ & $21.7 \pm 1.3$ & $22.5 \pm 5.0$ \\
$\quad$ MCP-1 & $15.9 \pm 2.3$ & $21.9 \pm 1.6$ & $21.2 \pm 3.5$ \\
Cholesterol $\left(\mathrm{mmol} \mathrm{l}^{-1}\right)$ & $11.2 \pm 3.2$ & $10.5 \pm 0.6$ & $11.2 \pm 0.5$ \\
\hline
\end{tabular}

ApoE-KO mice received PBS, lentiGFP or lenti35K at 6 weeks of age through hydrodynamic injection and were killed 3 months later. RANTES and MCP-1 were quantified in $50 \mu \mathrm{l}$ of mouse plasma using ELISA. Plasma cholesterol was assessed using an enzymatic assay. No significant differences were detected in either plasma chemokines or cholesterol concentrations between treatment groups ( $n=7-9$ mice/group).

atherosclerosis, we used lenti35K in ApoE-KO mice, and report the following important findings. First, lentiviral gene transfer with hydrodynamic delivery resulted in prolonged expression of soluble $35 \mathrm{~K}$ and GFP proteins in vivo in the ApoE-KO mouse for at least 3 months, confirmed by detection of both viral DNA and transgene mRNA in liver and soluble $35 \mathrm{~K}$ protein in the plasma. $35 \mathrm{~K}$ protein was not detected within transduced organs, on account of the efficient secretion of the $35 \mathrm{~K}$ protein from transduced cells, as we found previously. ${ }^{12} \mathrm{Im}-$ portantly, we have shown that hydrodynamic gene delivery using lentiviral vectors significantly reduces liver inflammation and necrosis compared with adenoviral delivery. Second, lentiviral 35K expression was sufficient to reduce plasma CC-CK activity for at least 3 months. Third, prolonged expression of $35 \mathrm{~K}$ protein significantly reduced both atherosclerotic plaque size and macrophage content in ApoE-KO mice. These results demonstrate that hydrodynamic lentiviral delivery of a soluble CC-CK inhibitor can achieve long-term inhibition of CC-CK activity sufficient to reduce atherosclerotic plaque formation. Furthermore, these studies suggest that the net effect of prolonged, broad-spectrum CC-CK inhibition is to inhibit macrophage recruitment and reduce atherosclerotic plaque progression.

Recombinant lentiviruses have emerged as useful tools to provide stable gene transfer and prolonged transgene expression, ${ }^{18,20}$ although the use of lentiviral vectors to target inflammatory pathways in atherosclerosis has not been evaluated previously. In this study, we now demonstrate that lentiviral gene delivery of $35 \mathrm{~K}$ can provide long-term CC-CK inhibition, leading to a significant reduction in aortic sinus atherosclerotic plaque size and plaque macrophage content. These results are a significant extension of earlier studies in which we used adenoviral-mediated gene transfer to express very high levels of $35 \mathrm{~K}$ to achieve a short-term CC-CK inhibition and a reduction in atherosclerotic plaque. ${ }^{13,23}$ The most striking differences between lentiviral and adenoviral 35K gene delivery are the level of protein expression achieved and the magnitude of vector-associated inflammation. Adenoviruses are able to transduce hepatocytes in vivo with a much higher efficiency than lentiviruses and therefore protein expression is significantly higher. ${ }^{24}$ Indeed, plasma from mice that had received adenovirus $35 \mathrm{~K}$ gene transfer contained more than 10 -fold higher levels of $35 \mathrm{~K}$ protein than lenti35K plasma. Nevertheless, the lower level of 

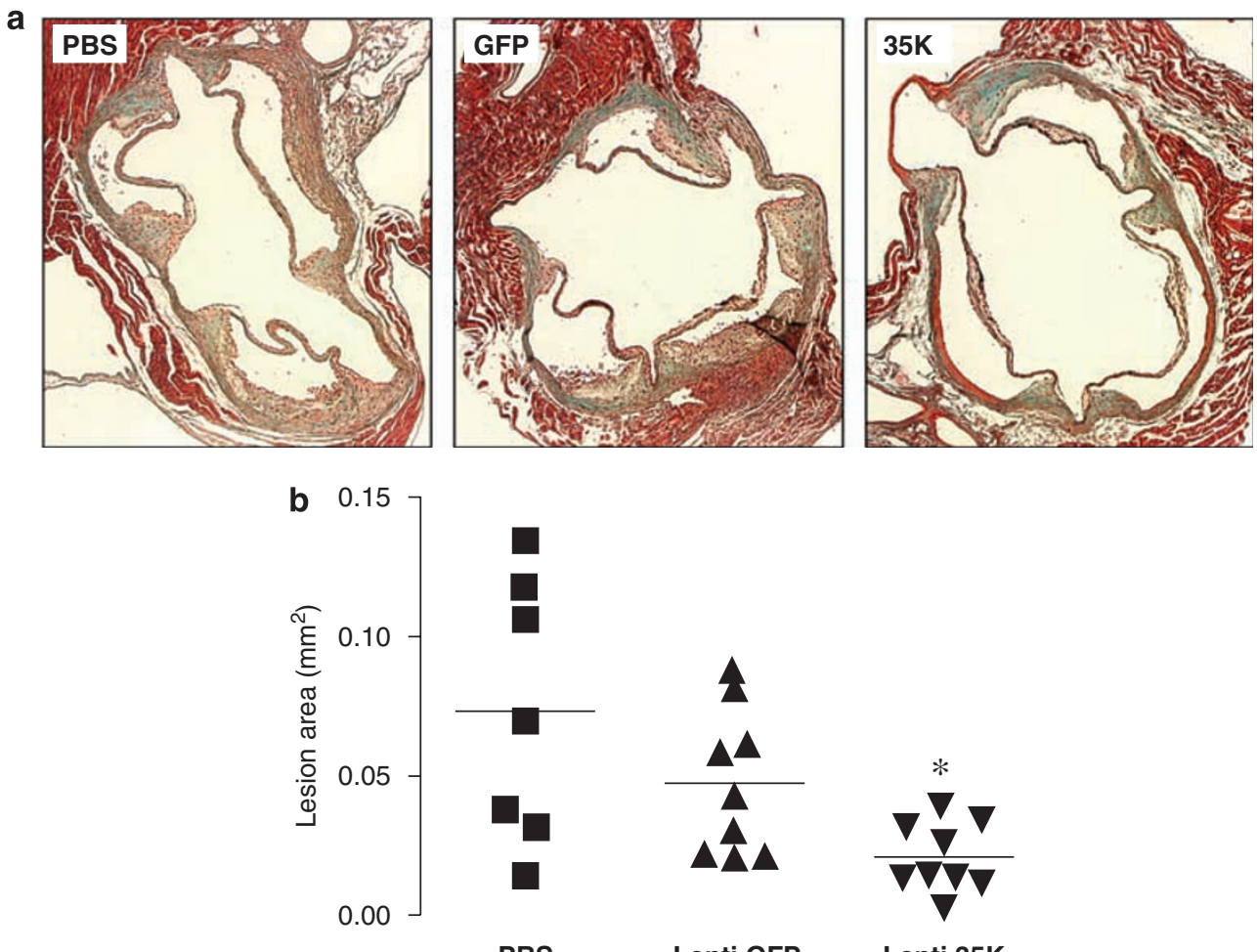

PBS

Lenti GFP

Lenti 35K

Figure 5 Long-term CC-chemokine inhibition with 35K reduces atherosclerosis in ApoE-KO mice. ApoE-KO mice received PBS, lentiGFP or lenti35K at 6 weeks of age through hydrodynamic injection and then were culled 3 months later. Total mean lesion area $\left(\mathrm{mm}^{2}\right)$ was determined from three paraffin-embedded sections per animal, taken from the base, centre and top of the aortic sinus, stained with MassonGoldner stain. (a) Representative lesions of aortic sinus sections in treatment groups. (b) Quantification of plaque lesion area $\left(\mathrm{mm}^{2}\right) .{ }^{*}$ Denotes a significant difference from PBS and lentiGFP control groups, $P<0.05, n=7-9$ mice/treatment group.

$35 \mathrm{~K}$ protein expression achieved with lenti35K, sustained over 3 months, was sufficient to reduce plasma CC-CK activity, reduce the progression of atherosclerosis and reduce macrophage recruitment into developing atherosclerotic plaques. These observations suggest that relatively low levels of $35 \mathrm{~K}$ protein, when sustained over more prolonged periods, can exert striking biological effects. These effects of $35 \mathrm{~K}$ gene delivery were achieved without the significant liver inflammation and necrosis that is typical of adenoviral gene delivery, characterized by leukocyte influx, and elevated serum ALT and AST levels.

We used hydrodynamic delivery to maximize the efficiency of lentiviral gene transfer in ApoE-KO mice. Hydrodynamic injection involves the rapid administration of a large volume of fluid, causing increased pressure in the liver that leads to the formation of pores on the membranes of the hepatocytes, facilitating vector entry. ${ }^{25-27}$ Previous studies have found that hydrodynamic injection is well tolerated in mice under general anesthesia and can result in hepatocyte transduction even by large DNA plasmids that would not normally be able to transduce cells in vivo. ${ }^{28-30}$ In preliminary experiments, we found that delivery of lentivirus through hydrodynamic injection significantly increased transduction efficiency compared with standard intravenous delivery and allowed a substantial reduction in virus particles to achieve comparable levels of gene transfer efficacy. Indeed, other studies have required up to 10 -fold more lentivirus particles to achieve sufficient biologically significant transduction. ${ }^{31-33}$ Thus, our study shows that hydrodynamic delivery is a useful technique to improve efficacy and reduce viral dose in lentiviral gene transfer. Along with the increase viral transduction following hydrodynamic delivery, the tropism of lentiviral vectors for the liver in vivo drives efficient production and release of the soluble $35 \mathrm{~K}$ molecule in this anatomical site.

35K blocks CC-CK activity by binding to key residues responsible for receptor activation and GAG binding. ${ }^{34}$ As such the CC-CKs are sequestered away from their receptors both being unable to actively bind to their receptor and also unable to immobilize on vascular surfaces to form a directional gradient. This will in turn prevent cells that would otherwise follow the CC-CK gradient into the atherosclerotic plaque from being able to do so. As a confirmation of this mechanism of action we observed that lenti35K significantly reduced CC-CK activity in the plasma, as judged by chemotaxis of CCR5 -transfected cells, but did not change the plasma concentration of the CC-CKs MCP-1 or RANTES, as measured by enzyme-linked immunosorbent assay (ELISA) (Table 1). This indicates that as expected $35 \mathrm{~K}$-bound chemokines are unable to provide a directional stimulus in a chemotaxis assay. This finding is also consistent with previous observations and suggests that CC-CKs bound to $35 \mathrm{~K}$ protein remain detectable by ELISA, but are functionally inactive. ${ }^{12}$

The results of our study provide important new insights into the role of chemokines in atherosclerosis. $35 \mathrm{~K}$ inhibits a broad range of CC-CKs. Some CC-CKs contribute to macrophage recruitment in atherosclerosis 
a
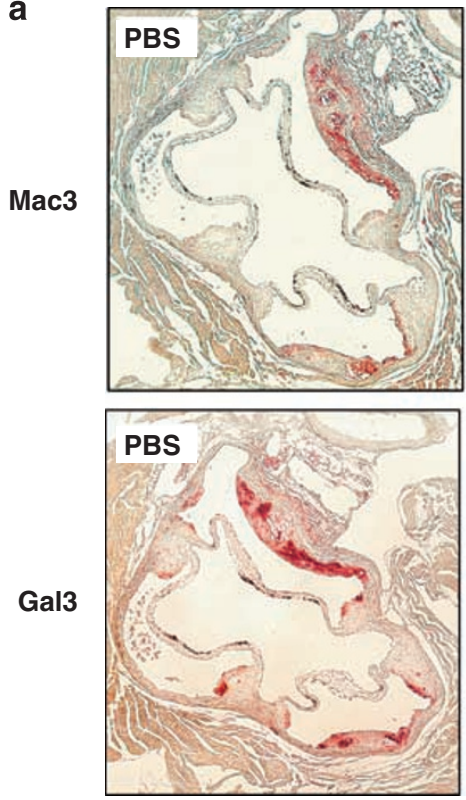
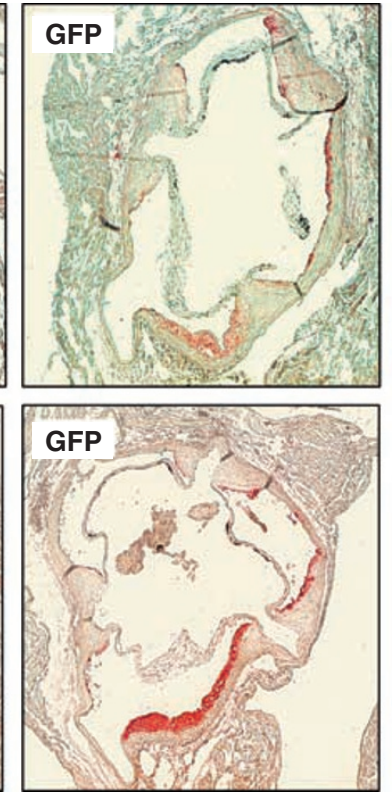
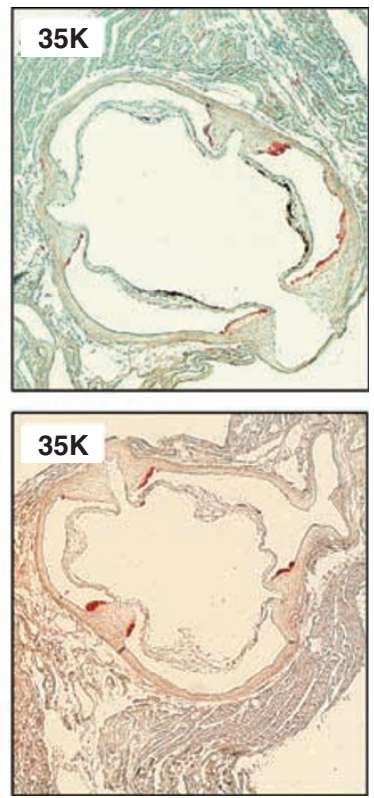

Gal3

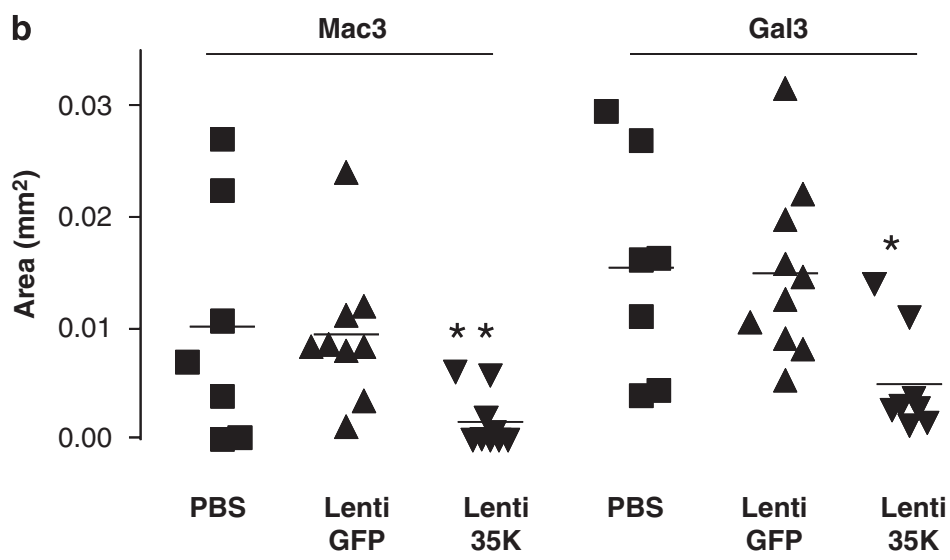

Figure 6 Long term CC-chemokine inhibition with 35K reduces macrophage content in atherosclerotic plaques of ApoE-KO mice. ApoE-KO mice received PBS, lentiGFP or lenti35K at 6 weeks of age through hydrodynamic injection and were killed 3 months later. Mac-3 antibody (1:50 dilution) and anti-Galectin-3 antibody (1:200 dilution) were used to independently detect lesion macrophages ( $\left.\mathrm{mm}^{2}\right)$ in sections from the centre of paraffin-embedded aortic sinus. (a) Representative images of mac-3 and gal3 staining from aortic sinus sections for each different treatment group. (b) Quantification of macrophage specific area $\left(\mathrm{mm}^{2}\right) .{ }^{* *}$ Denotes a significant difference from PBS and lentiGFP control groups, $P<0.01, n=7-9$ mice/treatment group. ${ }^{*}$ Denotes a significant difference from PBS and lentiGFP control groups, $P<0.05, n=7-9$ mice/treatment group.

(for example, MCP-1/CCL2, RANTES/CCL5), whereas others regulate macrophage emigration from plaques during plaque regression (for example, CCL19 and CCL21). ${ }^{14}$ The reduction of plaque progression by prolonged broad-spectrum CC-CK blockade suggests that the dominant or net effect of CC-CKs in atherosclerosis is towards plaque progression.

In conclusion, we demonstrate efficient lentiviral gene transfer of a recombinant CC-CK binding protein, $35 \mathrm{~K}$, through hydrodynamic injection in vivo in ApoE-KO mice. Lenti35K delivery provided prolonged 35K expression and inhibition of chemokine activity, showing that hydrodynamic delivery is a promising strategy to improve the efficiency of lentiviral gene transfer. Prolonged $35 \mathrm{~K}$ expression reduced both atherosclerotic plaque size and macrophage content, suggesting that long-term, broadspectrum CC-CK inhibition is a rational therapeutic strategy to inhibit atherosclerotic plaque development.

\section{Materials and methods}

Generation of recombinant lentivirus expressing $35 \mathrm{~K}$

A lentiviral transfer plasmid was constructed by cloning a fragment containing the $750 \mathrm{bp}$ fragment of $35 \mathrm{~K}$ of the Vaccinia virus (Lister strain), incorporating a carboxyterminal HA epitope tag, ${ }^{12}$ into pLenti6/V5-D-TOPO (Invitrogen, Pailey, UK). A recombinant lentivirus, lenti35K, was generated by co-transfecting the 293T17 cells with gag-pol, rev and VSV-G envelope $\left(\mathrm{PMD}_{2} \mathrm{G}\right)$ plasmids using an established protocol..$^{35} \mathrm{~A}$ control recombinant lentivirus, lentiGFP, encoding enhanced green fluorescence protein (Clontech, Mountain View, CA, USA) was prepared in parallel. Virus supernatants were harvested, filtered $(0.45 \mu \mathrm{m}$ pore size, low protein affinity) and concentrated by ultracentrifugation. Titer estimates were determined by p24 ELISA as $\sim 1 \times 10^{9}$ transducing units $(\mathrm{TU}) / \mathrm{ml}$. 
Transduction of Lenti35k and LentiGFP in vitro

Purified lentiviruses were added to 293 cells at a range of titers $\left(1 \times 10^{5}-1 \times 10^{1}\right)$ then incubated in the presence of polybrene (Sigma-Aldrich, Dorset, UK) $\left(8 \mu \mathrm{g} \mathrm{ml}^{-1}\right)$ for $24 \mathrm{~h}$ before changing to fresh media. Transduced cells were left to incubate for periods of up to 3 months. Media was collected and frozen periodically for subsequent detection of soluble $35 \mathrm{~K}$ protein using western blotting. Fluorescent cells transduced with LentiGFP were visualized under an inverted microscope and digital images were recorded (Nikon TE2000U).

\section{Animals and gene transfer}

ApoE-KO mice on a C57BL6 background (Jax Laboratories, ME, USA) were fed a regular chow diet for the duration of the experiment ( $n=7-9 /$ treatment group). At 6 weeks of age mice received either PBS or lentivirus by tail vein hydrodynamic injection $\left(7.5 \times 10^{7} \mathrm{TU}\right.$ diluted in $10 \% \mathrm{w} / \mathrm{v}$ body weight PBS, typically $2-2.5 \mathrm{ml}$ ), under isofluorane anesthesia. ${ }^{28}$

Twelve weeks following lentiviral delivery, mice were killed using an overdose of isofluorane anesthesia and plasma was collected by cardiac puncture. Mice were perfuzed with PBS through the left ventricle. Pieces of liver were collected and snap frozen for real-time PCR experiments. The femur, fibula and tibia were removed for collection of bone marrow. The heart and aorta were then perfusion-fixed using 4\% paraformaldehyde in PBS $(5 \mathrm{ml})$. Hearts, with aortic roots, were excised and fixed overnight in paraformaldehyde then embedded in paraffin. All animal procedures were carried out in accordance with the UK Home Office Animals (Scientific Procedures) Act 1986, and after local ethical review.

For toxicity experiments PBS or lentiGFP $\left(7.5 \times 10^{7} \mathrm{TU}\right.$ diluted in $10 \% \mathrm{w} / \mathrm{v}$ body weight PBS, typically $2-2.5 \mathrm{ml}$ ) were administered by hydrodynamic delivery as above, or AdGFP $\left(1 \times 10^{11} \mathrm{TU}\right.$ in $\left.0.3 \mathrm{ml}\right)$ by conventional i.v. injection. Mice were killed 1 week post gene transfer, with serum and liver samples being prepared as above.

\section{Liver histology}

Paraffin-embedded liver samples were sectioned $(7 \mu \mathrm{m})$ and stained with hematoxylin and eosin (Sigma), anti S100A9 antibody (a generous gift of Nancy Hogg, Cancer Research UK, London, UK) at $1 \mu \mathrm{g} \mathrm{ml}^{-1}$ or anti-CD3 antibody (DAKO) at 1:200. This was followed by a secondary biotinylated antibody (Vector Laboratories, UK) then avidin-biotin-AP complex and visualized with Vector Red alkaline phosphatase substrate (Vector Laboratories, UK).

\section{Serum transaminase measurements}

Liver enzymes were measured in serum samples using commercial enzymatic assays (Infinity ALT and AST liquid stable reagent, Thermo Scientific, Waltham, MA, USA) according to the manufacturer's instructions.

\section{Real-time RT-PCR for detection of lentivirus transgene expression}

Frozen liver samples (5 mg) were homogenized in Trizol. RNA was extracted and further purified using the RNeasy protocol (Qiagen, West Sussex, UK). RNA was reverse transcribed using Superscript II according to the manufacturer's instructions (Invitrogen). Taq-man cus- tom gene expression assays (FAM-labeled) were designed to quantify $35 \mathrm{~K}$ and GFP cDNA (35K-forward: GCTGATGGAAACACAGTGAATGC, reverse: CGGG AGAGTCCTTACCTTGTC, probe: ACGCTGGACAAT CTA, GFP-forward: GAGCGCACCATCTTCTTCAAG, reverse: TGTCGCCCTCGAACTTCAC, probe: ACGACG GCAACTACA). All real-time PCRs were performed using $100 \mathrm{ng}$ RNA equivalent. Gene expression data were normalized to $\alpha$-actin (housekeeping gene) and normalized to the highest expressing animal for each assay (GFP and $35 \mathrm{~K}$ ) using the $\Delta C_{\mathrm{t}}$ method. Reaction products from the $35 \mathrm{~K}$ assay were run on a $3 \%$ agarose gel and visualized using ethidium bromide.

\section{Real-time PCR for detection of viral DNA}

Frozen livers $(0.3 \mathrm{~g})$ were homogenized in extraction buffer (10 mM Tris- $\mathrm{HCl}$ pH $8.50 .45 \%$ Non-idet P40, $0.45 \%$ Tween-20, $0.5 \mathrm{mg} \mathrm{ml}^{-1}$ Proteinase K) and DNA was extracted using phenol/chloroform and re-suspended in TE buffer. All real-time PCRs were performed on $500 \mathrm{ng}$ of genomic DNA using SyberGreen I (BioRad, Hertfordshire, UK) in a $10 \mu \mathrm{l}$ reaction with $1 \mu \mathrm{M}$ of primers. Gene expression data was normalized to GAPDH (housekeeping gene) and expressed as relative to high expressing animal for each assay (GFP and 35K) using the $\Delta C_{\mathrm{t}}$ method. Reaction products from the $35 \mathrm{~K}$ assay were run on a 3\% agarose gel and visualized using ethidium bromide.

\section{Harvesting of bone marrow}

The femur, fibula and tibia were cleaned of all muscle tissue. The bone marrow was then flushed out using sterile PBS $(10 \mathrm{ml})$ with a needle $(26 \mathrm{G})$ and syringe $(10 \mathrm{ml})$. Cells were then re-suspended with a 19-G needle and passed through a cell strainer before plating out on $9 \mathrm{~cm}$ plates in RPMI-containing L-cell-conditioned media. Cells were left for $72 \mathrm{~h}$ before viewing and capturing digital images (Zeiss) under an inverted fluorescent microscope.

\section{Western immunoblotting}

To detect recombinant $35 \mathrm{~K}$ protein in cell media, 10-20 $\mu \mathrm{l}$ was separated on $14 \%$ SDS-PAGE gels. Following transfer to PVDF membranes, $35 \mathrm{~K}$ protein was detected using rabbit polyclonal anti-35K antibody diluted to 1:2000 (a generous gift from Peter Turner, University of Florida, Gainesville, FL, USA) followed by an anti-rabbit secondary antibody conjugated to horseradish peroxidase diluted 1:1500.

To evaluate $35 \mathrm{~K}$ in mouse plasma, $200 \mu \mathrm{l}$ of plasma was incubated overnight $\left(4^{\circ} \mathrm{C}\right)$ with monoclonal anti-HA agarose-conjugated beads (Sigma). Beads were washed, diluted 1:1 in $2 \times$ SDS sample buffer and proteins were denatured by heating at $95{ }^{\circ} \mathrm{C}$ for $3 \mathrm{~min}$. Beads were pelleted by centrifugation and the supernatant separated on $14 \%$ SDS-PAGE gels, transferred and immunoblotted as described above.

\section{Quantification of chemokine activity}

Specific CCR5 receptor-directed cell migration was assessed using transwell membranes $(6.0 \mathrm{~mm}$ diameter, $8 \mu \mathrm{m}$ pore size, Receptor Technologies, Banbury, UK) as described earlier. ${ }^{12}$ Briefly, 293 cells were grown to $50 \%$ confluence in Dulbecco's modified Eagle's media $(\mathrm{DMEM})+10 \%(\mathrm{v} / \mathrm{v})$ fetal calf serum, then co-transfected 
(Fugene6, Roche, Hertfordshire, UK) with plasmids encoding CCR5 and EGFP to facilitate visualization. Transfected cells were harvested and allowed to migrate overnight towards mouse plasma $(10 \mu \mathrm{l})$ placed in the lower chamber. Migrated cells on the underside of membranes were fixed and quantified by computer analysis of EGFP fluorescence in confocal microscope images. Each experimental sample was analyzed in duplicate, and three separate images were quantified for each membrane.

\section{Histology and immunohistochemistry of atherosclerosis in aortic root sections}

Serial transverse sections $(7 \mu \mathrm{m})$ of the paraffin-embedded hearts were taken through the aortic root. Serial sections were collected from the whole of the aortic root for which all three valve cusps were visible. To quantify lesion area, 3 sections were chosen from base, middle and top of the aortic root, being $80 \mu \mathrm{m}$ apart across a standardized $160 \mu \mathrm{m}$ area. They were stained with Masson-Goldner/elastin stain (VWR international, Leicestershire, UK) for evaluation of atherosclerotic plaque size $\left(\mathrm{mm}^{2}\right)$. For evaluation of macrophage plaque content, paraffin sections were stained with mouse macrophage antigen Mac-3 (Pharmingen, Oxford, UK, rat anti-mouse)at a $1: 50$ dilution overnight $\left(4{ }^{\circ} \mathrm{C}\right)$ or antiGalectin 3 (R\&D Systems, Abingdon, UK) at 1:200 for $2 \mathrm{~h}$ at room temperature. This was followed by a secondary anti-rat or anti-goat biotinylated antibody, respectively (Vector Laboratories, Peterborough, UK) then avidinbiotin-AP complex and visualized with Vector Red alkaline phosphatase substrate (Vector Laboratories). Sections ( $n=3 /$ mouse) were quantified from digitized microscopic images using Image Pro-Plus software (Media Cybernetics, Bethesda, MD, USA).

\section{Detection of plasma chemokines in mouse plasma}

Mouse RANTES and MCP-1 CC-CKs were detected in mouse plasma $(50 \mu \mathrm{l})$ using ELISA kits (Quantikine, R\&D systems) following the manufacturer's instructions.

\section{Plasma lipids}

Plasma lipids were measured using an enzymatic assay (Roche) on heparinized blood plasma using a Cobas Mira Plus automated analyzer (Roche).

\section{Statistical analysis}

All values are expressed as the mean \pm s.e.m. Data were analyzed using a one-way analysis of variance and the Tukeys post hoc test of significance, comparing the controls to the treatment groups. A value of $P<0.05$ was the criterion of significance.

\section{Acknowledgements}

We gratefully acknowledge the kind donation of rabbit polyclonal $35 \mathrm{~K}$ antibody from Peter Turner and Dick Moyer, Department of Molecular Genetics and Microbiology, University of Florida. We gratefully acknowledge the kind donation of rat monoclonal S100A9 antibody (2B10) from Nancy Hogg, Cancer Research UK, London Research Institute, UK. We thank the Lentiviral Vector Production Unit of the Swiss Institute of Technology Lausanne (EPFL), supported by the
Association Française contre les Myopathies (AFM), for the production of the lentivirus used in the in vivo studies. We thank Ashley Hale for assistance with the liver transaminase assays. This work, in the laboratories of DRG and KMC, was supported by the British Heart Foundation.

\section{References}

1 Bursill CA, Channon KM, Greaves DR. The role of chemokines in atherosclerosis: recent evidence from experimental models and population genetics. Curr Opin Lipidol 2004; 15: 145-149.

2 Glass CK, Witztum JL. Atherosclerosis. the road ahead. Cell 2001; 104: 503-516.

3 Wilcox JN, Nelken NA, Coughlin SR, Gordon D, Schall TJ. Local expression of inflammatory cytokines in human atherosclerotic plaques. I Atheroscler Thromb 1994; 1: S10-S13.

4 Haley KJ, Lilly CM, Yang JH, Feng Y, Kennedy SP, Turi TG et al. Overexpression of eotaxin and the CCR3 receptor in human atherosclerosis: using genomic technology to identify a potential novel pathway of vascular inflammation. Circulation 2000; 102: 2185-2189.

5 Reape TJ, Rayner K, Manning CD, Gee AN, Barnette MS, Burnand KG et al. Expression and cellular localization of the CC chemokines PARC and ELC in human atherosclerotic plaques. Am J Pathol 1999; 154: 365-374.

6 Greaves DR, Hakkinen T, Lucas AD, Liddiard K, Jones E, Quinn $\mathrm{CM}$ et al. Linked chromosome 16q13 chemokines, macrophagederived chemokine, fractalkine, and thymus- and activationregulated chemokine, are expressed in human atherosclerotic lesions. Arterioscler Thromb Vasc Biol 2001; 21: 923-929.

7 Boring L, Gosling J, Cleary M, Charo IF. Decreased lesion formation in CCR2-/ - mice reveals a role for chemokines in the initiation of atherosclerosis. Nature 1998; 394: 894-897.

8 Gosling J, Slaymaker S, Gu L, Tseng S, Zlot CH, Young SG et al. MCP-1 deficiency reduces susceptibility to atherosclerosis in mice that overexpress human apolipoprotein B. J Clin Invest 1999; 103: 773-778.

9 Braunersreuther V, Zernecke A, Arnaud C, Liehn EA, Steffens S, Shagdarsuren E et al. Ccr5 but not Ccr1 deficiency reduces development of diet-induced atherosclerosis in mice. Arterioscler Thromb Vasc Biol 2007; 27: 373-379.

10 Burns JM, Dairaghi DJ, Deitz M, Tsang M, Schall TJ. Comprehensive mapping of poxvirus vCCI chemokine-binding protein. Expanded range of ligand interactions and unusual dissociation kinetics. J Biol Chem 2002; 277: 2785-2789.

11 Smith CA, Smith TD, Smolak PJ, Friend D, Hagen H, Gerhart M et al. Poxvirus genomes encode a secreted, soluble protein that preferentially inhibits beta chemokine activity yet lacks sequence homology to known chemokine receptors. Virology 1997; 236: 316-327.

12 Bursill C, Cai S, Channon KM, Greaves DR. Adenoviralmediated delivery of a viral chemokine binding protein blocks CC-chemokine activity in vitro and in vivo. Immunobiology 2003; 207: 1-10.

13 Bursill CA, Choudhury RP, Ali Z, Greaves DR, Channon KM. Broad-spectrum CC-chemokine blockade by gene transfer inhibits macrophage recruitment and atherosclerotic plaque formation in apolipoprotein E-knockout mice. Circulation 2004; 110: $2460-2466$.

14 Trogan E, Feig JE, Dogan S, Rothblat GH, Angeli V, Tacke F et al. Gene expression changes in foam cells and the role of chemokine receptor CCR7 during atherosclerosis regression in ApoE-deficient mice. Proc Natl Acad Sci USA 2006; 103: 3781-3786. 
15 Kobayashi H, Carbonaro D, Pepper K, Petersen D, Ge S, Jackson H et al. Neonatal gene therapy of MPS I mice by intravenous injection of a lentiviral vector. Mol Ther 2005; 11: 776-789.

16 Carbonaro DA, Jin X, Petersen D, Wang X, Dorey F, Kil KS et al. In vivo transduction by intravenous injection of a lentiviral vector expressing human ADA into neonatal ADA gene knockout mice: a novel form of enzyme replacement therapy for ADA deficiency. Mol Ther 2006; 13: 1110-1120.

17 Bot I, Guo J, Van Eck M, Van Santbrink PJ, Groot PH, Hildebrand $\mathrm{RB}$ et al. Lentiviral shRNA silencing of murine bone marrow cell CCR2 leads to persistent knockdown of CCR2 function in vivo. Blood 2005; 106: 1147-1153.

18 Kohn D. Lentiviral vectors ready for prime-time. Nat Biotechnol 2007; 25: 65-66.

19 Ren B, O’Brien BA, Swan MA, Koina ME, Nassif N, Wei MQ et al. Long-term correction of diabetes in rats after lentiviral hepatic insulin gene therapy. Diabetologia 2007; 50: 1910-1920.

20 Amado RG, Chen ISY. Lentiviral vectors-the promise of gene therapy within reach? Science 1999; 285: 674-676.

21 Hobbs JA, May R, Tanousis K, McNeill E, Mathies M, Gebhardt $\mathrm{C}$ et al. Myeloid cell function in MRP-14 (S100A9) null mice. Mol Cell Biol 2003; 23: 2564-2576.

22 Papaspyridonos M, McNeill E, de Bono JP, Smith A, Burnand KG, Channon KM et al. Galectin-3 is an amplifier of inflammation in atherosclerotic plaque progression through macrophage activation and monocyte chemoattraction. Arterioscler Thromb Vasc Biol 2008; 28: 433-440.

23 Ali ZA, Bursill CA, Hu Y, Choudhury RP, Xu Q, Greaves DR et al. Gene transfer of a broad spectrum CC-chemokine inhibitor reduces vein graft atherosclerosis in apolipoprotein E-knockout mice. Circulation 2005; 112: I235-I241.

24 St George JA. Gene therapy progress and prospects: adenoviral vectors. Gene Therapy 2003; 10: 1135-1141.

25 Crespo A, Peydro A, Dasi F, Benet M, Calvete JJ, Revert F et al. Hydrodynamic liver gene transfer mechanism involves transient sinusoidal blood stasis and massive hepatocyte endocytic vesicles. Gene Therapy 2005; 12: 927-935.
26 Kobayashi N, Nishikawa M, Hirata K, Takakura Y. Hydrodynamics-based procedure involves transient hyperpermeability in the hepatic cellular membrane: implication of a nonspecific process in efficient intracellular gene delivery. J Gene Med 2004; 6: 584-592.

27 Zhang G, Gao X, Song YK, Vollmer R, Stolz DB, Gasiorowski JZ et al. Hydroporation as the mechanism of hydrodynamic delivery. Gene Therapy 2004; 11: 675-682.

28 Hibbitt OC, Harbottle RP, Waddington SN, Bursill CA, Coutelle C, Channon KM et al. Delivery and long-term expression of a $135 \mathrm{~Kb}$ LDLR genomic DNA locus in vivo by hydrodynamic tail vein injection. J Gene Med 2007; 9: 488-497.

29 Zhang G, Song YK, Liu D. Long-term expression of human alpha1-antitrypsin gene in mouse liver achieved by intravenous administration of plasmid DNA using a hydrodynamics-based procedure. Gene Therapy 2000; 7: 1344-1349.

30 Liu F, Song Y, Liu D. Hydrodynamics-based transfection in animals by systemic administration of plasmid DNA. Gene Therapy 1999; 6: 1258-1266.

31 De Palma M, Venneri MA, Naldini L. In vivo targeting of tumor endothelial cells by systemic delivery of lentiviral vectors. Hum Gene Ther 2003; 14: 1193-1206.

32 Lamikanra A, Myers KA, Ferris N, Mitrophanous KA, Carroll MW. In vivo evaluation of an EIAV vector for the systemic genetic delivery of therapeutic antibodies. Gene Therapy 2005; 12: 988-998.

33 Bao L, Jaligam V, Zhang XY, Kutner RH, Kantrow SP, Reiser J. Stable transgene expression in tumors and metastases after transduction with lentiviral vectors based on human immunodeficiency virus type 1. Hum Gene Ther 2004; 15: 445-456.

34 Zhang L, Derider M, McCornack MA, Jao SC, Isern N, Ness T et al. Solution structure of the complex between poxvirusencoded CC chemokine inhibitor vCCI and human MIP-1beta. Proc Natl Acad Sci USA 2006; 103: 13985-13990.

35 Klages N, Zufferey R, Trono D. A stable system for the high-titer production of multiply attenuated lentiviral vectors. Mol Ther 2000; 2: 170-176. 